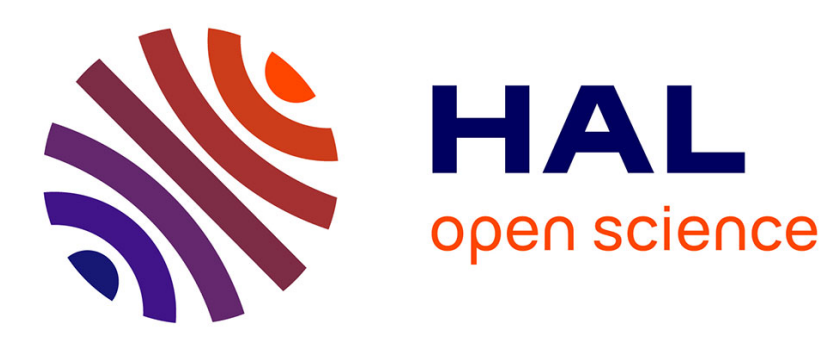

\title{
Solitons as a Possible Probe for Detecting Defects in Solids
}

\author{
S. Ozawa, H. Tanaka, Y. Hiki
}

\section{To cite this version:}

S. Ozawa, H. Tanaka, Y. Hiki. Solitons as a Possible Probe for Detecting Defects in Solids. Journal de Physique IV Proceedings, 1996, 06 (C8), pp.C8-841-C8-844. 10.1051/jp4:19968181 . jpa-00254618

\section{HAL Id: jpa-00254618 https://hal.science/jpa-00254618}

Submitted on 1 Jan 1996

HAL is a multi-disciplinary open access archive for the deposit and dissemination of scientific research documents, whether they are published or not. The documents may come from teaching and research institutions in France or abroad, or from public or private research centers.
L'archive ouverte pluridisciplinaire HAL, est destinée au dépôt et à la diffusion de documents scientifiques de niveau recherche, publiés ou non, émanant des établissements d'enseignement et de recherche français ou étrangers, des laboratoires publics ou privés. 


\title{
Solitons as a Possible Probe for Detecting Defects in Solids
}

\author{
S. Ozawa, H. Tanaka* and Y. Hiki** \\ Applied Physics Group, Faculty of Engineering, Ibaraki University, Nakanarusawa, Hitachi 316, Japan \\ * NTT Data Communications Systems Co., 5-10-24 Nishikasai, Edogawa-ku, Tokyo 134, Japan \\ ** Faculty of Science, Tokyo Institute of Technology, Emeritus, 39-3-303 Motoyoyogi, Shibuya-ku, \\ Tokyo 151, Japan
}

\begin{abstract}
Molecular dynamics computer simulation has been performed for solitons and phonons propagating in one- and two-dimensional anharmonic mass-spring model crystals. An input pulse displacement was applied to atoms in a crystal, and propagating phonons or solitons were produced when the input pulse was smaller or larger. Collisions of the solitons and phonons with mass, spring constant, and anharmonicity defects were studied to obtain the energy transmission coefficients for collisions. Collision characteristics were different for solitons and phonons.
\end{abstract}

\section{INTRODUCTION}

Phonon or ultrasound is conveniently used to investigate defects in crystals or flaws in materials. We intend to study whether solitons propagating in solids can also be used as another probe for that purpose. We have started computer simulation experiments on phonons using the molecular dynamics (MD) method. A one-dimensional mass-spring model crystal with lattice anharmonicity up to the fourth order in the atomic potential was used. The basic properties of propagating acoustic phonons and conducting thermal phonons were simulated [1]. Phonon conduction in crystals containing mass defects was then studied $[2,3]$. The study was extended to the simulation of propagation of solitons in model crystals [4]. In this study, solitons accompanied by phonons were generated, the stability of the solitons against mutual collision was observed, and the dependence of the propagation velocity of solitons on the lattice anharmonicity was investigated. Then, collision of a soliton with lattice defects in crystals was simulated [5-7]. Three kinds of defect were taken into consideration: mass defect, spring constant defect and anharmonicity defect. Now the study is extended to the case of two-dimensional crystal, and a preliminary report on this subject is given.

\section{METHOD}

In our computer experiments, the molecular dynamics (MD) method is used for one-dimensional (1D) and two-dimensional (2D) mass-spring model crystals. First, the case of $1 \mathrm{D}$ crystal is explained. The model crystal is in the form of a circular chain. A cyclic boundary condition is satisfied for atomic vibration of the model crystal. The atomic mass, interatomic spacing, and number of atoms in the crystal are $m, L$, and $N$. For the interatomic force constants, anharmonic potential up to the fourth order, or anharmonic force up to the third order, is taken. The equation of motion for the $i$ th atom in the crystal is

$$
\begin{aligned}
m\left(\mathrm{~d}^{2} D_{i} / \mathrm{d} T^{2}\right)= & -C^{(1)}\left(D_{i}-D_{i-1}\right)-C^{(2)}\left(D_{i}-D_{i-1}\right)^{2}-C^{(3)}\left(D_{i}-D_{i-1}\right)^{3} \\
& +C^{(1)}\left(D_{i+1}-D_{i}\right)+C^{(2)}\left(D_{i+1}-D_{i}\right)^{2}+C^{(3)}\left(D_{i+1}-D_{i}\right)^{3},
\end{aligned}
$$

where $D_{i}$ is the atomic displacement, $C^{(j)}$ is the $j$ th-order interatomic force constant, and $T$ represents time. The ratio of the force constants $C^{(1)}: C^{(2)}: C^{(3)}$ is appropriately chosen such that the magnitudes of the terms in RHS of eq. (1) become successively smaller. The equations for $N$ atoms are numerically integrated using the fourth-order Runge-Kutta method [8], and the displacement and velocity of atoms are determined. In the simulation, a system of MD units is used: $m=1$, 
$L=1000, T=1$, and $C^{(1)}=1$. When the above MD units are adopted, the minimum period of the atomic vibration is $T_{0}=3.14$. Further, a discrete time interval (MD steps) is taken, and in the computation $1 \mathrm{MD}$ step $=0.05 \mathrm{MD}$ unit.

The procedure of simulation for the 2D crystal is essentially the same as in the $1 \mathrm{D}$ case. An $N \times N$ square lattice crystal is taken, nearest neighbor central forces up to the third order of anharmonicity are considered. Cyclic boundary conditions are given both for the $x$ - and $y$-directions. In the computation, MD step with shorter time interval, which is $1 / 100$ of those used in the $1 D$ case, are adopted because of the high dimensionality.

\section{RESULTS}

\subsection{Production of solitons}

The method of introducing solitons into the model crystal is illustrated. In the computation of the ID case, the model crystal used is composed of $N=128$ atoms, and the ratios of the force constants (in MD units) are here arbitrarily chosen as $C^{(2)} / C^{(1)}=-0.5$ and $C^{(3)} / C^{(1)}=0.25$. Two neighboring atoms at the positions $X=63$ and 64 are suddenly displaced in the same direction by an amount $D_{\mathrm{p}}$ (in MD units), and displacements of all atoms successively occur. Figure 1(a) shows snapshots of atomic displacement $D$ (arbitrary units) vs atomic position $X(=0-128)$ at time $T$ (MD steps)
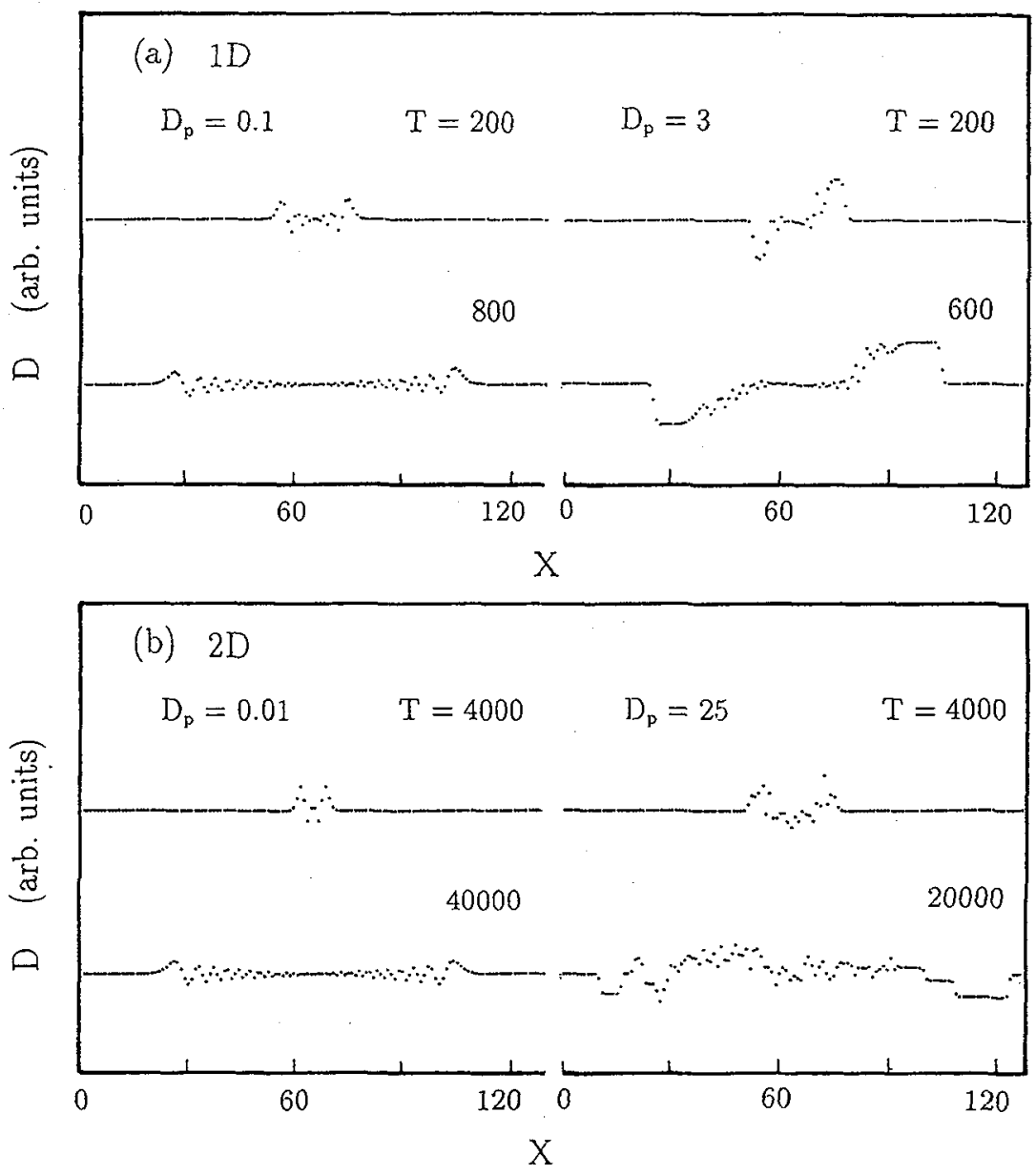

Figure 1: Atomic displacement $D$ vs atomic position $X$ at time $T$ after applying pulse input $D_{\mathrm{p}}$ showing phonon (small $D_{\mathrm{p}}$ ) and soliton (large $D_{\mathrm{p}}$ ) cases, for one-dimensional (a) and twodimensional (b) crystals. 
after pulsed displacements are applied to the two atoms. When the initial displacement $D_{\mathrm{p}}$ is small, for example $D_{\mathrm{p}}=0.1$, wave packets propagating in opposite directions are excited. These waves are composed of phonons. When the displacement $D_{\mathrm{p}}$ is large enough, for example $D_{\mathrm{p}}=3$, solitons propagating in opposite directions can be produced. Note that the atomic displacement curve for a soliton is kink-shaped, as can be seen in the figure. There are also tails following the solitons, which are composed of phonons. The displacements of two solitons are opposite in their signs. Solitons are produced only when the atomic displacements in the crystal are large enough, since in this case the crystal anharmonicity is effectively large. Thus we can excite solitons or phonons in the crystal by using larger or smaller input displacement. By observing the stability or instability for mutual collisions, we can identify the excitations as solitons or phonons [4].

In the case of $2 \mathrm{D}$ crystal, the number of atoms is $N_{x}=N_{y}=128$. The force constants are the same as in the $1 \mathrm{D}$ case. All atoms on two atomic rows $X=63$ and $X=64$ are suddenly displaced in the $x$ direction by $D_{\mathrm{p}}$, and movements of the atoms on the atomic row $Y=64$ are observed. Figure 1(b) shows the snapshot of atomic displacement in the $x$ direction of atoms at the position $X$ at time $T$. Note that the MD steps chosen are shorter than those in the $1 D$ case, so that the apparent values of $T$ are larger. In the $2 \mathrm{D}$ simulation, the obtained results are almost the same as in the $1 \mathrm{D}$ case. Phonon packets or solitons with phonon tails propagating in the opposite directions are produced for small or large input pulse. In this case, the displacements of two solitons are the same in their signs. It is important that propagating solitons can thus successfully be produced also in two-dimensional model crystals.

\subsection{Collision with defects}

The problem is treated for the $1 \mathrm{D}$ case in the following manner. In these more quantitative computations, the ratios of the force constants (in $\mathrm{MD}$ units) were chosen as $C^{(2)} / C^{(1)}=-0.1$ and $C^{(3)} / C^{(1)}=0.01$. By adopting these choices, the ratio of the second-, third-, and fourth-order elastic constants of the crystal become as $C_{\mathrm{IJ}}: C_{\mathrm{IJK}}: C_{\mathrm{IJKL}}=1:-10: 100$ [7], which is consistent with situations in actual crystals [9]. The number of atoms in the crystal is 600, and a defect is put at the position $X=400$. Two atoms at $X=299$ and 300 are displaced by $D_{\mathrm{p}}$, and the excitations, solitons or phonons, propagating in the positive direction are noted. The time variation of the total energy $E$ ( $=$ kinetic + potential) in the part of the crystal between $X=400$ and 500 is computed. We call this part of the crystal "observing crystal part" (OCP).

In the case of soliton propagation in an ideal (defect-free) crystal, the energy in the OCP rapidly increases when the soliton arrives at the edge of the OCP. When the soliton propagates out of the OCP, the energy rapidly decreases. This is because the energy of a soliton is sharply concentrated in space. The energy increment in the OCP is diminished when a defect is introduced at the edge of the OCP. This is originated from the reflection of soliton by the defect. The energy transmission coefficient $T_{E}$ can be calculated from the ratio of the energy increments in the OCP for the cases of defect and ideal crystals [7].

In the case of propagating phonons, the energy in the OCP gradually increases and then decreases when the phonons pass through the OCP. This is because the energy of a phonon packet is not spatially concentrated, and successively coming and going phonons gradually increase and then decrease the energy in the OCP. Even in this case the transmission coefficient $T_{E}$ can be obtained by using adequately averaged values of the energy increments in the OCP [7].

The results of the computed energy transmission coefficient $T_{\mathrm{E}}$ are as follows. Figure $2(\mathrm{a})$ is for the case of a mass defect, where an atom with mass $m^{\prime}$ different from those of body atoms $m$ is introduced into the crystal, and the mass ratio $\mathrm{m}^{\prime} / m$ is varied over a wide range. Results for different input displacements $D_{\mathrm{p}}$ are shown, and the smaller and the larger $D_{\mathrm{p}}$ corresponds to the phonon and soliton cases, respectively. Figure $2(b)$ is for a spring constant defect, where the first-order force constant, or the spring constant, on both sides of an atom is changed to $C^{(1) t}$ from the spring constant $C^{(1)}$ for body atoms, and the ratio $C^{(1) \prime} / C^{(1)}$ is widely varied. Figure $2(c)$ is for an anharmonicity defect, where the second-order anharmonic force constant is changed to $C^{(2) r}$ from $C^{(2)}$ on both sides of an atom. The ratio $C^{(2) \prime} / C^{(2)}$ is not changed so widely because of the restriction of series expansion in eq. (1). In the cases of mass defect and spring constant defect, the energy transmission $T_{\mathrm{E}}$ decreases or the energy reflection increases as the degree of disorder due to the defect (for example, deviation of $\mathrm{m}^{\prime} / \mathrm{m}$ from unity) is enhanced for both phonons and solitons. In the case of anharmonicity defect, most noticeable is that phonons are not reflected and solitons are reflected by the defect. This is understandable because the crystal anharmonicity is essentially 

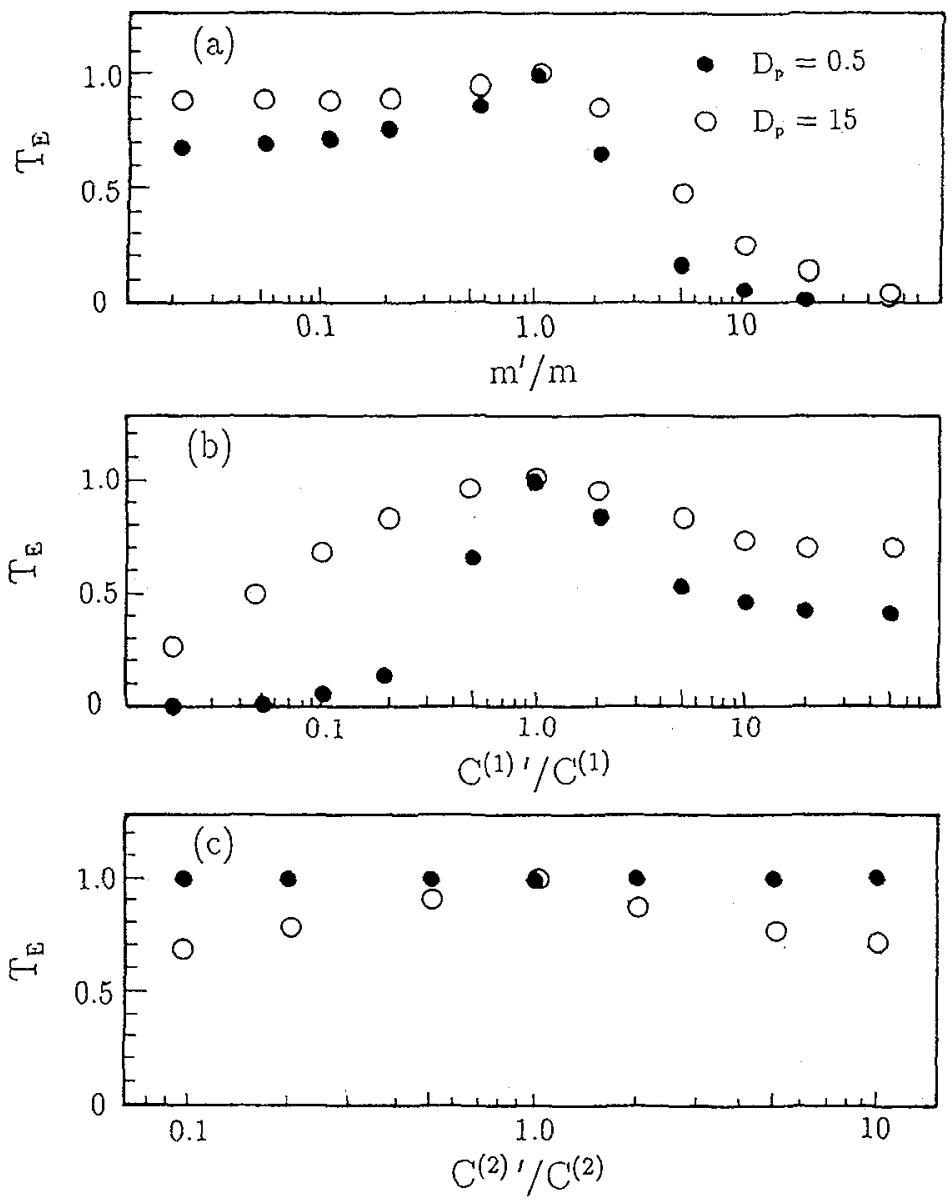

Figure 2: Energy transmission coefficients $T_{E}$ for (a) mass defect, (b) spring constant defect, and (c) anharmonicity defect.

important for solitons.

There can be defects in crystals where the mass, spring constant, and anharmonicity are unusual. These defects can be distinguished using both phonons and solitons as probes. The anharmonicity defect in particular can be sensitively detected by solitons. Furthermore, a soliton is spatially concentrated, which is convenient when the soliton is used as a probe since the resolution power can be much great. In this context, we are now developing the study of two-dimensional crystal case.

\section{References}

[1] Ozawa S. and Hiki Y., Jpn. J. Appl. Phys. 30, Suppl. 30-1 (1991) 46-48.

[2] Hiki Y. and Ozawa S., Proc. Int. Conf. on Phonon Scattering in Condensed Matter VII (Springer, Berlin, 1993) p. 19-21.

[3] Ozawa S. and Hiki Y., J. Alloys \& Compd. 211/212 (1994) 471-474.

[4] Ozawa S. and Hiki Y., Jpn. J. Appl. Phys. 34 (1995) 2590-2595.

[5] Ozawa.S. and Hiki Y., Physica B 219 \& 220 (1996) 473-475.

[6] Hiki Y. and Ozawa S., Proc. 14th Int. Symp. on Nonlinear Acoustics (Nanjing Univ., Nanjing, 1996) 381-386.

[7] Ozawa S. and Hiki Y., Jpn. J. Appl. Phys. (1996) in press.

[8] Henrici P., Elements of Numerical Analysis (Wiley, New York, 1964).

[9] Hiki Y., Ann. Rev. Mater. Sci. 11 (1981) 51-73. 